university of Graz (Styria, Austria). They stated that it is not admissible to deduce the amount and duration a local effect from the half life elimation in another compartement. On the other side the most relevant parameter to measure the efficiency of such an intervention is pain. Two of the studies mentioned in our report (Senel et al., Dehkordi et al.) clearly stated an improvement in the amount and duration of the analgetic effect by the supplement of tramadol. The first idea to add an opioid for our nerve root infiltrations was the report of several patients that they experienced a return of their radicular pain after about half a day after the infiltration an then a slow decrease of this pain over the next couple of days. From our experience so far this rebound of pain has decreased since we add tramadol to our infiltration regime. Unfortunately we don't have the data to support this observation. We also agree that one would not expect a benefit from tramadol after 2 weeks but the relatively low risk of adding tramadol argues for further investigation concerning the pain relief in the

\section{Reference}

1. Murthy BV, Pandya KS, Booker PD, Murray A, Lintz W, Terlinden R. Pharmacokinetics of tramadol in children after i.v. or caudal epidural administration. Br J Anaesth 2000; 84:346-349. first 24 to 48 hours.

The response to your second objection is quite easy. The specification for the amount of ropivacain should have read $5 \mathrm{mg}$ instead of $2 \mathrm{mg}$. We apologize for that error.

Mathias Wewalka MD, MSc

Department of Physical Medicine and Rehabilitation Landesklinikum

Mistelbach, Austria.

E-mail: mathias.wewalkamistelbach.lknoe.at

Ahmadollah Abdelrahimsai, MD

Department of Physical Medicine and Rehabilitation Sanatorium Hera

Vienna, Austria ,

Gunther Wiesinger, MD

Associate Professor

University Hospital Salzburg

Paracelsus Medical University

Salzburg, Austria.

Eva Maria Uher, MD

Departmento f Physical Medicine and Rehabilitation Landesklinikum

Mistelbach, Austria.

\title{
Muscle Rigidity Associated with Pregabalin
}

Pregabalin, like gabapentin, is a structural analog of gamma aminobutyric acid (GABA), which binds to the alpha-2-delta subunit of N-type calcium channels, resulting in decreased release of several neurotransmitters $(1,2)$. It has been shown to be effective for neuropathic pain, but pregabalin can induce adverse events such as dizziness, somnolence, and peripheral edema (3). Other side effects have been rarely reported. Two patients who developed muscle rigidity while taking oral pregabalin are presented.

Case 1 was a 69-year-old man who presented with pain and numbness in his right upper and right lower limbs. He had a traumatic right brachial plexus injury 10 years earlier. He was prescribed clonazepam (4 mg orally), etodolac (400 mg orally), and gabapentin (1600 mg orally) for pain. However, the visual analog scale (VAS) score for pain at rest was $66 \mathrm{~mm}$. Therefore, he was changed to pregabalin (300 mg orally) from gabapentin (1600 mg orally) when pregabalin was approved in Japan in October 2010. The VAS score at rest was 59 $\mathrm{mm}$, and his pain decreased. Seven days after starting pregabalin, he felt rigidity in his arms and legs. Pregabalin (300 mg orally) was changed to gabapentin (1600 mg orally) 14 days after starting pregabalin. The muscle rigidity thereafter resolved.

In the second case, a 75-year-old man presented with a chief concern of sharp and burning pain in the 7th and 8th dermatomes of the right chest following thoracic postherpetic neuralgia 10 years earlier. He was prescribed oxycodone (10 mg orally) for pain. However, the VAS score at rest was $82 \mathrm{~mm}$. In addition, he was prescribed pregabalin (75 $\mathrm{mg}$ irally); the VAS score at rest was $42 \mathrm{~mm}$, and his pain decreased. However, because he felt that his leg muscles were stiff and rigid, 
he returned to our pain clinic 8 days after starting pregabalin. His serum creatine phophokinase was $76 \mathrm{IU} / \mathrm{L}$ (normal range for men, 67-251 IU/L), his blood urea nitrogen was $19 \mathrm{mg} / \mathrm{dL}$ (normal range, 8-20 mg/dL), and his serum creatinine was as low as $0.76 \mathrm{mg} / \mathrm{dL}$ (normal range, 0.8-1.2 $\mathrm{mg} / \mathrm{dL}$ ). There was no evidence of renal failure or rhabdomyolysis. Pregabalin (75 mg orally) was changed to clonazepam (1.5 mg orally); his muscle stiffness and rigidity resolved, and his VAS score at rest was between $30 \mathrm{~mm}$ and $40 \mathrm{~mm}$.

Pregabalin and gabapentin are some of the newer drugs approved as anticonvulsants and for the treatment of neuropathic pain (4), and they are classified as adjuvant drugs in the World Health Organization analgesic ladder. However, compared to gabapentin, pregabalin is more rapidly absorbed (one hour for pregabalin, 3-4 hours for gabapentin) and has higher bioavailability (more than $90 \%$ for pregabalin, 33-66\% for gabapentin) (5). The absorption of gabapentin is saturable, whereas the absorption of pregabalin increases with increasing dose, resulting in linear pharmacokinetics (6). Pregabalin has the advantage of requiring less frequent dosing and has been as effective as gabapentin at much lower doses. However, adverse events associated with pregabalin have been reported frequently, though muscle rigidity after pregabalin administration has not.

Perez Lloret and colleagues (7) reported a case of parkinsonism after pregabalin administration. In their case, the patient developed a parkinsonian-like syndrome with rigidity in both upper and lower limbs. Their report, however, does not suggest a possible mechanism for muscle rigidity induced by pregabalin.

On the other hand, pregabalin is a calcium blocker. Martí Massó and colleagues (8) reported calcium blocker-induced parkinsonism in patients older than 60 . We thought that the muscle rigidity was a symptom of parkinsonism, and the mechanism of muscle rigidity probably involved reduction of the release and synthesis of striatal dopamine by a calcium blocker effect (9). Moreover, it has been reported that calcium blockers reduce presynaptic levels of dopamine in the brain and may precipitate parkinsonian symptoms (10).

The second potential reason for muscle rigidity after taking pregabalin may be rhabdomyolysis, since muscle rigidity is a symptom of rhabdomyolysis. $\mathrm{Pa}$ tients with renal failure may be particularly prone to developing muscle rigidity. Pregabalin is eliminated by the kidneys, and $98 \%$ of absorbed doses is excreted unchanged in the urine. Therefore, one would expect that the blood concentration of pregabalin would be high and adverse effects would occur frequently in patients with renal failure. Although the present cases were older patients and may have had decreased kidney function, neither of them had renal failure.

In summary, muscle rigidity in patients taking normal doses of pregabalin was described. To the best of our knowledge, this is the first report describing muscle rigidity in patients due to pregabalin treatment.

Yuka Matsuki, MD

Department of Anesthesiology \& Reanimatology University of Fukui, Faculty of Medicine Sciences 23-3 Matsuokashimoaizuki, Eiheijicho, Yoshidagun Fukui 910-1193, Japan

E-mail: ymatsuki@u-fukui.ac.jp

Mari Tabata MD

Department of Anesthesiology \& Reanimatology University of Fukui

Faculty of Medicine Sciences, Fukui, Japan

E-mail: mari@gd5.so-net.ne.jp

Yasunari Nobukawam, MD

Department of Anesthesiology \& Reanimatology, University of Fukui

Faculty of Medicine Sciences

Fukui, Japan

tainobu@u-fukui.ac.jp

Mikako Sakai, MD

Department of Anesthesiology

Kano Hospital

Fukui, Japan

E-mail: tmsakai@wd5.so-net.ne.jp

Yoshikazu Yasuda, MD

Department of Anesthesiology \& Reanimatology

University of Fukui

Faculty of Medicine Sciences

Fukui, Japan

E-mail: yyasuda@u-fukui.ac.jp

Maki Mizogam, MD

Department of Anesthesiology \& Reanimatology

University of Fukui

Faculty of Medicine Sciences, Fukui, Japan

E-mail: makikai@u-fukui.ac.jp 
Kenji Shigemi, MD

Department of Anesthesiology \& Reanimatology

University of Fukui

Faculty of Medicine Sciences

Fukui, Japan

E-mail: kshigemi@u-fukui.ac.jp

\section{References}

1. Rosenstock J, Tuchman M, LaMoreaux L, Sharma U. Pregabalin for the treatment of painful diabetic peripheral neuropathy: A double-blind, placebo-controlled trial. Pain 2004; 110:628-638.

2. Smith HS, Harris R, Clauw D. Fibromyalgia: An afferent processing disorder leading to a complex pain generalized syndrome. Pain Physician 2011; 14:E217E245.

3. Freynhagen R, Strojek K, Griesing $T$, Whalen E, Balkenohl M. Efficacy of pregabalin in neuropathic pain evaluated in a 12-week, randomized, double-blind, multicentre, placebo-controlled trial of flexible-and fixed-dose regimens. Pain
2005; 115:254-263.

4. Gilron I. Gabapentin and pregabalin for chronic neuropathic and early postsurgical pain: Current evidence and future directions. Curr Opin Anaesthesiol 2007; 8. 20:456-472.

5. Bockbrader HN, Wesche D, Miller R, Chapel S, Janiczek N, Burger P. A comparison of the pharmacokinetics and 9 . pharmacodynamics of pregabalin and gabapentin. Clin Pharmacokinet 2010; 49:661-669.

6. Hellwig S, Amtage F. Pregabalin- induced cortical negative myoclonus in a patient with neuropathic pain. Epilepsy Behav 2008; 13:418-420.
7. Perez Lloret S, Amaya M, Merello $M$. Pregabalin-induced parkinsonism: A case report. Clin Neuropharmacol 2009; 32:353-354.

8. Martí Massó JF, Obeso JA, Carrera N, Martínez-Lage JM. Aggravation of Parkinson's disease by cinnarizine. J Neurol Neurosurg Psychiatry 1987; 50:804-805.

9. Pileblad E, Carlsson A. In vivo effects of the Ca2+-antagonist nimodipine on dopamine metabolism in mouse brain. J Neural Transm 1986; 66:171-187.

10. Terland O, Flatmark T. Drug-induced parkinsonism: Cinnarizine and flunarizine are potent uncouplers of the vacuolar $\mathrm{H}+-$ ATPase in catecholamine storage vesicles. Neuropharmacology 1999; 3.

\section{Caudal Epidural Injections for the Treatment of Tarlov Cysts: Suggestions for the Better Results}

\section{To THE EDITOR}

It is with great interest that we read the case report by Freidenstein et al, "Minimally invasive interventional therapy for Tarlov cysts causing symptoms of interstitial cystitis," published in the 2012 March/April issue of Pain Physician (1).

This is a well organized and prepared case report which showed successful result in treating Tarlov cysts with symptoms similar to interstitial cystitis. To treat these cysts in this article, authors performed caudal epidural injections by inserting the needle only a few millimeters through the sacral hiatus and administering $3 \mathrm{ml}$ of $1 \%$ lidocaine and $40 \mathrm{mg}$ of triamcinolone. Authors thought that the reduction of inflammation around the cysts may lessen the cyst distention and subsequent pressure on the nearby nerve root. In ad- dition, they recommended to avoid dural puncture during the procedure and to use low-volume drugs to prevent inadvertent increase in pressures. We applaud the authors' work, however, we have some different points of view on the injection method and the volume of injectate.

First, we believe that the injection method should be adjusted according to the location and size of Tarlov cysts. Because the cyst itself or the secondary inflammation hinders the delivery of steroid and local anesthetic agent to the target lesion, it would be more effective to inject as near as possible to the cysts. In addition, Tarlov cysts possibly cause symptoms compressing or irritating nearby nerve roots as the herniated intervetebral discs (2). Also, caudal epidural injections need large volume 